\title{
Associations between Lifetime Adversity and Obesity Treatment in Patients with Morbid Obesity
}

\author{
Reidun Rønningen ${ }^{a} \quad$ Anne Cathrine Parelius Wammer ${ }^{a}$ \\ Nina Holte Grabner ${ }^{b}$ Tone Gretland Valderhaug ${ }^{a}$ \\ ${ }^{a}$ Department of Endocrinology, Akershus University Hospital HF, Lørenskog, Norway; \\ ${ }^{b}$ Department of Psychiatry, Unit for Consultation-Liason Psychiatry, Akershus University \\ Hospital, Lørenskog, Norway
}

\author{
Keywords \\ Bariatric surgery · Eating behavior - Obesity $\cdot$ Psychological aspects $\cdot$ Stress overnutrition
}

\begin{abstract}
Background: Bariatric surgery is associated with greater and more sustainable weight loss compared with lifestyle intervention programs. On the other hand, bariatric surgery may also be associated with physical and psychosocial complications. The influence of psychological evaluation on treatment choice, however, is not known. We aimed to examine variables associated with treatment choice and, specifically, if self-reported lifetime adversity influenced obesity treatment, i.e. bariatric surgery, high-intensive lifestyle treatment or low-intensive lifestyle treatment in primary care. Methods: We consecutively included 924 patients from the registry study of patients with morbid obesity at Akershus University Hospital, Lørenskog, Norway. Treatment selection was made through a shared decision-making process. Self-reported lifetime adversity was registered by trained personnel. Logistic regression models were used to assess the associations between obesity treatment and possible predictors. $\boldsymbol{R e}$ sults: Patients who chose bariatric surgery were more likely to have type 2 diabetes (DM2) compared with patients who chose lifestyle treatment (bariatric surgery: $35 \%$, high-intensive lifestyle treatment: $26 \%$, and low-intensive lifestyle treatment: $26 \% ; p=0.035$ ). Patients who chose bariatric surgery were less likely than patients who chose lifestyle intervention to report lifetime adversity (bariatric surgery: 39\%, high-intensive lifestyle treatment: $47 \%$, and lowintensive lifestyle treatment: $51 \% ; p=0.004$ ). After multivariable adjustments, increasing BMI, having DM2, and joint pain were associated with choosing bariatric surgery over non-surgical
\end{abstract}


obesity treatment (odds ratio [95\% Cl]: BMI 1.03 [1.01-1.06], DM2 1.47 [1.09-1.99], and joint pain 1.46 [1.08-1.96]). Self-reported lifetime adversity was furthermore associated with lower odds of choosing bariatric surgery in patients with morbid obesity (0.67 [0.51-0.89]). Conclusion: This study shows that increasing $\mathrm{BMI}, \mathrm{DM} 2$, and joint pain were all associated with treatment choice for obesity. In addition, self-reported lifetime adversity was associated with the patients' treatment choice for morbid obesity. Consequently, we suggest that decisions concerning obesity treatment should include dialogue-based assessments of the patients' lifetime adversity.

(C) 2019 The Author(s)

Published by S. Karger AG, Basel

\section{Introduction}

Obesity is mostly measured by means of BMI, but anthropometric classification systems do not reflect the presence or severity of comorbidities [1]. Increasing BMI is associated with increased risk of type 2 diabetes (DM2), cardiovascular disease, and incidence of several types of cancers [2]. Bariatric surgery is associated with sustainable weight loss and reduced morbidity and mortality in patients with morbid obesity [3]. However, a recent cohort study of 1,888 patients with morbid obesity showed that patients treated with bariatric surgery had increased risk of complications such as abdominal pain, gastrointestinal ulcers, and iron deficiency compared with patients who were treated with lifestyle treatment after 6.5 years of follow-up [4]. This study furthermore showed a 50\% increased risk of new-onset depression in patients who underwent bariatric surgery compared with patients who received intensive lifestyle treatment [4]. Moreover, reduced alcohol tolerability is considered a complication from bariatric surgery, and empirical evidence indicates that gastric bypass increases the risk of developing both alcohol use disorders and substance use disorders [5, 6]. One study showed that alcohol use disorder more than doubled from $7 \%$ prior to surgery to $16 \% 7$ years after surgery [5].

An obesity classification system applied in a retrospective population study was superior to BMI in predicting mortality [7]. Nevertheless, BMI and DM2 remain the predominant predictors of bariatric surgery as obesity treatment [8]. The importance of building healthy therapeutic relations between patients and healthcare providers was demonstrated in a recent study that assessed patient perspectives in the role of primary care in obesity management [9]. Also, another recently published study revealed divergence in perception and attitudes between patients with obesity and HCP's perspective of obesity treatment [10]. Although assessments of psychological issues are considered essential for the identification of the optimal treatment choice at an individual level, the influence of an individual's psychological health on obesity treatment is not known. The aim of this study was to assess the impact of self-reported lifetime adversity on the subsequent treatment choice for morbid obesity.

\section{Material and Methods}

Design and Study Population

This cross-sectional study was conducted at a secondary specialist care center in the southeast region of Norway from January 1, 2014 until December 15, 2017. A total of 1,160 treatment-seeking patients with morbid obesity referred from general practice were eligible for study participation. The flow chart of the study population is presented in Figure 1. Out of 1,007 patients who signed an informed consent, we excluded 80 patients with missing data (treatment choice: $n=37$, lifetime adversity: $n=49$, treatment choice and lifetime adversity combined: $n=6$ ) and 3 patients with BMI under 35, leaving 924 patients with morbid 
Rønningen et al.: Lifetime Adversity Predicts Treatment Choice for Morbid Obesity

Fig. 1. Flowchart of the study

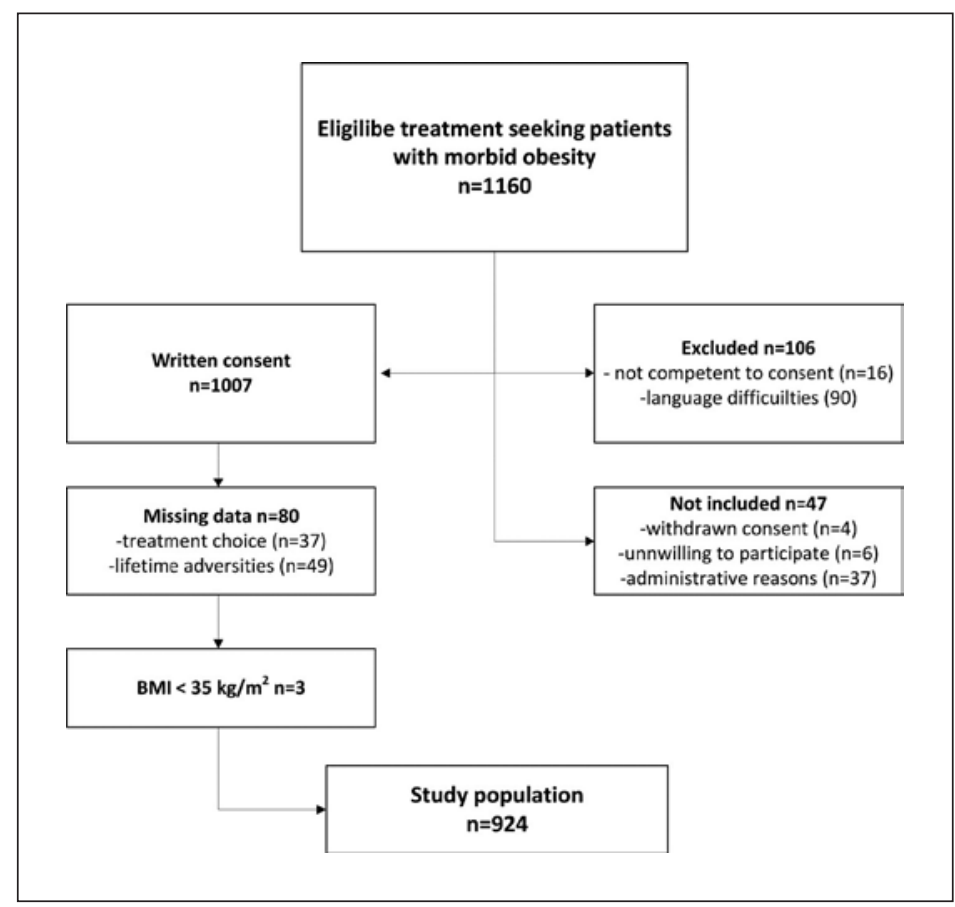
population.

obesity to be included in the final study. The study was approved by the Regional Committee for Medical and Health Research Ethics (2013/2042). The study was performed in accordance with the Declaration of Helsinki [11].

\section{Definitions}

Lifetime adversity was defined in patients with self-reported experiences during childhood or adolescence (such as maltreatment, abuse, neglect, parental alcohol or drug abuse, parental incarceration, parental psychiatric illness, parental inter-partner violence, parental death) or during adulthood (such as marginalization, emotional traumatized or physical violation of any kind) or if they had previously been diagnosed with post-traumatic stress disorder (PTSD). Patients who used psychoactive drugs at baseline were defined as having psychiatric illness. Patients who had a prior history of DM2 or a fasting plasma glucose $\geq 7.0$ $\mathrm{mmol} / \mathrm{L}$ or an $\mathrm{HbA1c} \geq 6.5 \%$ were defined as having DM2. Metabolic syndrome was defined according to the joint interim statement of the International Diabetes Federation Task Force on Epidemiology and Prevention; National Heart, Lung and Blood Institute; American Heart Association; World Heart Federation; International Atherosclerosis Society; and International Association for the Study of Obesity [12].

\section{Data Collection}

Data were retrieved in a clinical setting by trained personnel. Patients had their weight and height measured wearing light clothing, without shoes, and BMI was subsequently calculated $\left(\mathrm{kg} / \mathrm{m}^{2}\right)$. Blood pressure was measured with an appropriate cuff after at least 5 min rest with the patient seated in an upright position. Three measurements were registered and the average of the second and the third measurement was used in the study. The following data were assessed in dialog with the patients: employment status, use of medication, onset and history of obesity and obesity related comorbidities such as diabetes, hypertension, and joint pain. Patients were furthermore asked about their mental health, categorized as having selfreported anxiety or depression (yes/no). Information on lifetime adversity (yes/no) and psychiatric history was retrieved through dialog with the patients combined with searching the medical records when available. The investigators furthermore explored eventually adverse experiences prior to the onset of weight gain, and whether these might have influenced eating habits, physical activities, social life, or emotional reactions. For example, the patients were asked about adverse experiences or if bad or stressful feelings might have contributed to emotional eating and subsequent weight gain. The study outcome was the patients' choice of obesity treatment made together with trained personnel in a shared decision-making process, i.e. bariatric 
Rønningen et al.: Lifetime Adversity Predicts Treatment Choice for Morbid Obesity

surgery, high-intensive lifestyle treatment, or low-intensive lifestyle treatment in primary care. Bariatric surgery is not performed at our secondary center for morbid obesity. Patients who were ambivalent towards bariatric surgery or who disagreed with the treatment decision, were offered a second evaluation at a surgical center to help with the final decision for obesity treatment.

\section{Statistical Analysis}

Proportions are reported as numbers with percent and continuous variables as mean \pm standard deviation (SD). Differences between the treatment groups were analyzed using Pearson's chi-square test for categorical data and one-way analysis of variance or Kruskal-Wallis H test for continuous data. Post hoc analyses for comparison between two groups were performed by Student $t$ test for continuous data. Correlations were calculated with Pearson's correlation coefficient. Assumptions of linearity were assessed by the Box-Tidwell test. The goodness of fit was tested using the Hosmer-Lemeshow test. The overlap indices and the Cohen's kappa coefficient of agreement $(\kappa)$ for self-reported lifetime adversity and self-reported anxiety or depression as well as between lifetime adversity and psychiatric illness were calculated. Obesity treatment (i.e. bariatric surgery, high-intensive lifestyle treatment, and low-intensive lifestyle treatment) was modeled as dependent variable with lifetime adversity as the primary explanatory variable. A backward logistic regression approach was used to identify possible confounders. Variables with $p$ values below 0.10 were included in the final model (Wald test). As a result, the following variables were removed from the final model: age (years), gender, duration of obesity (years), self-reported anxiety or depression, and physical inactivity. The following variables were included in the final multivariable models: BMI, DM2, employment, joint pain, and lifetime adversity. Multivariable logistic regression analysis was used to assess the associations between lifetime adversity and obesity treatment. The analysis was furthermore stratified by gender.

The analyses were performed using IBM SPSS Statistics, version 25.0 (SPSS Inc., Chicago, IL, USA). $p$ values less than 0.05 were considered statistically significant.

\section{Results}

Overall $46 \%$ of the population had experienced lifetime adversity, and there was a significant difference between female and male gender in the prevalence of self-reported lifetime adversity ( $51 \%$ vs. $35 \%, p<0.001)$. Patients' characteristics according to treatment choice are presented in Table 1. Patients opting for bariatric surgery were more likely to have DM2 compared with the patients who chose lifestyle treatment (bariatric surgery 35\%, highintensive lifestyle treatment $26 \%$, and low-intensive lifestyle treatment $26 \% ; p=0.035$ ). Compared with patients who chose non-surgical treatment, patients opting for bariatric surgery were more likely to report joint pain (bariatric surgery vs. low-intensive lifestyle treatment: $35 \%$ vs. $28 \% ; p=0.027$; bariatric surgery vs. high-intensive lifestyle treatment: $35 \%$ vs. $27 \%$; $p=0.029$ ). There was a significant difference in self-reported lifetime adversity across obesity treatment choices. Patients who chose bariatric surgery were less likely than patients who opted for non-surgical treatment (i.e. low-intensive lifestyle treatment or highintensive lifestyle treatment) to report lifetime adversity (bariatric surgery 39\%, highintensive lifestyle treatment 47\%, and low-intensive lifestyle treatment 51\%; $p=0.004$ ) (Table 1). Also, patients who chose low-intensive lifestyle treatment were less likely than those who chose high-intensive lifestyle treatment or bariatric surgery to be employed (bariatric surgery 66\%, high-intensive lifestyle treatment $74 \%$, and low-intensive lifestyle treatment 56\%; $p<0.001$ ) (Table 1).

A total of $23(3 \%)$ patients reported eating disorders out of which 17 patients also reported lifetime adversity. The overlap between self-reported anxiety or depression and self-reported lifetime adversity is presented in Figure 2. A total of 477 (52\%) patients with morbid obesity were categorized with either self-reported anxiety or depression or with selfreported lifetime adversity. There was a fair overlap between self-reported anxiety or 
Fig. 2. The Venn diagram shows the overlap between self-reported anxiety or depression and selfreported lifetime adversity.

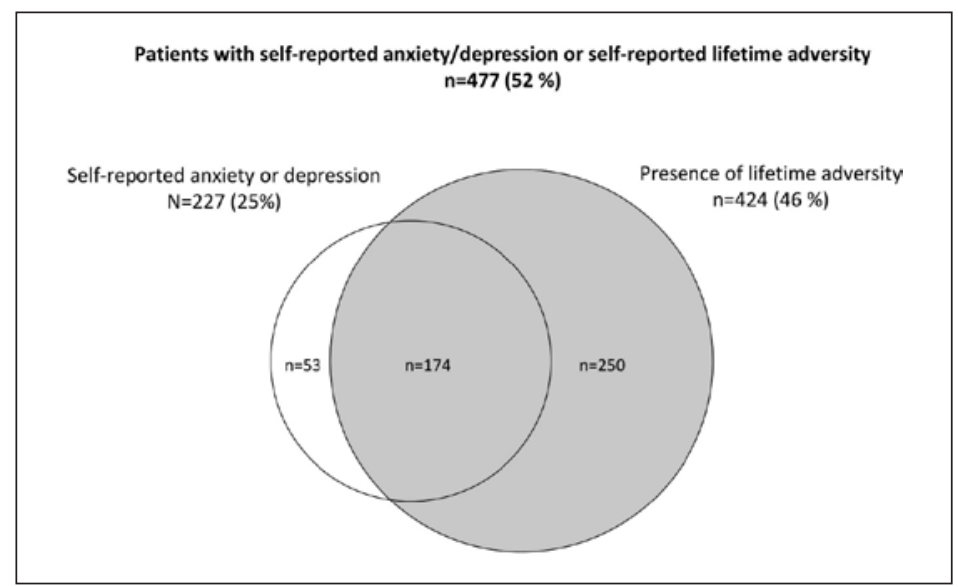

Table 1. Patients' characteristics by obesity treatment

\begin{tabular}{|c|c|c|c|c|c|}
\hline & Total & $\begin{array}{l}\text { Low-intensive } \\
\text { lifestyle } \\
\text { treatment }\end{array}$ & $\begin{array}{l}\text { High-intensive } \\
\text { lifestyle } \\
\text { treatment }\end{array}$ & $\begin{array}{l}\text { Bariatric } \\
\text { surgery }\end{array}$ & $p$ value \\
\hline Patients, $n$ & 924 & 398 & 198 & 328 & - \\
\hline Age (SD), years* & 45 (17) & 44 (18) & 45 (13) & 45 (14) & 0.621 \\
\hline Female gender, $n(\%)$ & $617(67 \%)$ & $265(67 \%)$ & $128(65 \%)$ & $224(68 \%)$ & 0.687 \\
\hline Body weight (SD), kg & $130(22)$ & $129(21)$ & $132(23)$ & $131(22)$ & 0.247 \\
\hline BMI & $44.5(5.7)$ & $44.1(5.3)$ & $44.5(5.9)$ & $45.0(5.9)$ & 0.089 \\
\hline Obesity class, BMI & & & & & 0.322 \\
\hline $35-40$ & $189(21 \%)$ & $87(22 \%)$ & $43(22 \%)$ & $59(18 \%)$ & \\
\hline $40-50$ & $587(64 \%)$ & $258(65 \%)$ & $122(62 \%)$ & 207 (64\%) & \\
\hline$\geq 50$ & $146(16 \%)$ & $53(13 \%)$ & $33(17 \%)$ & $60(18 \%)$ & \\
\hline Duration of obesity (SD), years* & $21(16)$ & $20(16)$ & $22(16)$ & $22(15)$ & 0.014 \\
\hline Systolic blood pressure (SD), mm Hg & $140(43)$ & $138(19)$ & $143(86)$ & 139 (19) & 0.366 \\
\hline Diastolic blood pressure (SD), mm Hg & $77(11)$ & $76(11)$ & $78(10)$ & $77(12)$ & 0.114 \\
\hline Metabolic syndrome, $n(\%)$ & $586(64 \%)$ & $245(62 \%)$ & $127(64 \%)$ & $214(65 \%)$ & 0.514 \\
\hline Type 2 diabetes, $n(\%)$ & $270(29 \%)$ & $105(26 \%)$ & $52(26 \%)$ & $113(35 \%)$ & 0.035 \\
\hline Hypertension, $n(\%)$ & $559(61 \%)$ & $230(58 \%)$ & $131(66 \%)$ & $198(60 \%)$ & 0.144 \\
\hline Employment, $n(\%)$ & $582(63 \%)$ & $222(56 \%)$ & $145(74 \%)$ & $215(66 \%)$ & $<0.001$ \\
\hline Joint pain, $n(\%)$ & $280(30 \%)$ & $112(28 \%)$ & $53(27 \%)$ & $115(35 \%)$ & 0.062 \\
\hline Physical inactivity, $n(\%)$ & $752(85 \%)$ & $328(85 \%)$ & $163(85 \%)$ & $261(85 \%)$ & 0.982 \\
\hline Family history of diabetes, $n(\%)$ & $279(30 \%)$ & $114(29 \%)$ & $52(26 \%)$ & $113(35 \%)$ & 0.094 \\
\hline Family history of CVD, $n(\%)$ & $291(32 \%)$ & $109(27 \%)$ & $65(33 \%)$ & 117 (35\%) & 0.052 \\
\hline Family history of obesity, $n(\%)$ & $359(39 \%)$ & $135(34 \%)$ & $79(40 \%)$ & $145(44 \%)$ & 0.017 \\
\hline Anxiety or depression, $n(\%)$ & $227(25 \%)$ & $103(26 \%)$ & $49(25 \%)$ & $75(23 \%)$ & 0.642 \\
\hline Psychiatric illness, $n(\%)$ & $111(12 \%)$ & $42(11 \%)$ & $23(12 \%)$ & $46(14 \%)$ & 0.352 \\
\hline Lifetime adversity, $n(\%)$ & $424(46 \%)$ & $204(51 \%)$ & $92(47 \%)$ & $128(39 \%)$ & 0.004 \\
\hline
\end{tabular}

Data are presented as mean (SD) or median (interquartile range) and proportions $(n[\%])$ * Denotes comparison between groups with Kruskal-Wallis $\mathrm{H}$ test for non-parametric data.

depression and self-reported lifetime adversity (overlap index $=0.365$ and $\kappa=0.316 ; p<$ 0.0029), whereas the overlap between lifetime adversity and psychiatric illness was poor (overlap index $=0.161$ and $\kappa=0.116 ; p<0.023$ ).

The odds ratios (OR) for the shared decision of obesity treatment are shown in Table 2 and Figure 3. After multivariable adjustments, BMI, DM2, and joint pain were associated with 
Rønningen et al.: Lifetime Adversity Predicts Treatment Choice for Morbid Obesity

Table 2. Variables associated with treatment choice in patients with morbid obesity

\begin{tabular}{|c|c|c|c|c|c|c|}
\hline & \multicolumn{2}{|c|}{$\begin{array}{l}\text { Low-intensive } \\
\text { lifestyle treatment }\end{array}$} & \multicolumn{2}{|c|}{$\begin{array}{l}\text { High-intensive } \\
\text { lifestyle treatment }\end{array}$} & \multicolumn{2}{|c|}{ Bariatric surgery } \\
\hline & OR & $95 \%$ CI & OR & $95 \%$ CI & OR & $95 \%$ CI \\
\hline \multicolumn{7}{|l|}{ All patients } \\
\hline BMI & 0.97 & $0.95,0.99$ & 1.00 & $0.97,1.03$ & 1.03 & $1.01,1.06$ \\
\hline DM2 & 0.74 & $0.55,0.99$ & 0.90 & $0.63,1.29$ & 1.47 & $1.09,1.99$ \\
\hline Employment & 0.55 & $0.41,0.73$ & 1.85 & $1.29,2.66$ & 1.24 & $0.92,1.67$ \\
\hline Joint pain & 0.74 & $0.55,1.00$ & 0.91 & $0.63,1.30$ & 1.46 & $1.08,1.96$ \\
\hline Lifetime adversity & 1.35 & $1.03,1.77$ & 1.11 & $0.80,1.53$ & 0.67 & $0.51,0.89$ \\
\hline \multicolumn{7}{|l|}{ Female gender } \\
\hline BMI & 0.96 & $0.93,0.99$ & 1.01 & $0.98,1.05$ & 1.03 & $1.00,1.06$ \\
\hline DM2 & 0.68 & $0.45,1.01$ & 0.90 & $0.55,1.48$ & 1.58 & $1.06,2.35$ \\
\hline Employment & 0.52 & $0.37,0.74$ & 2.21 & $1.40,3.49$ & 1.16 & $0.81,1.66$ \\
\hline Joint pain & 0.62 & $0.43,0.90$ & 1.02 & $0.66,1.58$ & 1.59 & $1.10,2.28$ \\
\hline Lifetime adversity & 1.32 & $0.94,1.84$ & 1.34 & $0.90,2.01$ & 0.61 & $0.44,0.87$ \\
\hline \multicolumn{7}{|l|}{ Male gender } \\
\hline BMI & 0.98 & $0.94,1.03$ & 0.97 & $0.92,1.02$ & 1.04 & $1.00,1.09$ \\
\hline DM2 & 0.79 & $0.49,1.27$ & 0.83 & $0.48,1.44$ & 1.49 & $0.91,2.43$ \\
\hline Employment & 0.59 & $0.36,0.98$ & 1.25 & $0.68,2.31$ & 1.52 & $0.88,2.63$ \\
\hline Joint pain & 1.07 & $0.64,1.80$ & 0.74 & $0.39,1.40$ & 1.17 & $0.68,2.01$ \\
\hline Lifetime adversity & 1.55 & $0.95,2.52$ & 0.79 & $0.44,1.42$ & 0.74 & $0.44,1.26$ \\
\hline
\end{tabular}

DM2, type 2 diabetes. The odds ratios (ORs) with 95\% confidence interval (CI) are presented for all patients and stratified by gender.

increased OR of choosing bariatric surgery as obesity treatment, whereas self-reported lifetime adversity was associated with lower OR of choosing bariatric surgery (OR [95\% CI]: BMI 1.03 [1.01-1.06], DM2 1.47 [1.09-1.99], joint pain 1.46 [1.08-1.96], and lifetime adversity 0.67 [0.51-0.89]). Compared with patients who did not report lifetime adversity, patients with self-reported lifetime adversity were associated with 35\% increased OR of choosing low-intensive lifestyle treatment (1.35 [1.03-1.77]). Moreover, being employed was associated with a two-fold increased OR choosing high-intensive lifestyle treatment compared with patients who were unemployed (1.85 [1.29-2.66]).

\section{Discussion}

The main and novel finding of this study of 924 patients with morbid obesity was that the prevalence of lifetime adversity is high among patients with morbid obesity and that there is an association between self-reported lifetime adversity and the shared decision-making process of obesity treatment in patients with morbid obesity. Although it is well established that implementing a preoperative psychological assessment is important to prevent psychological complications of bariatric surgery, there are no standard protocols to help health professionals to implement this assessment $[13,14]$. A previous study reported that preoperative cognitive behavioral therapy was not effective in the preparation of patients awaiting bariatric surgery [15]. Although one study has reported improved mental health after bariatric surgery, there is no consensus on therapeutic consequences of psychological evaluation [16]. Thus, the presentstudy underscores the importance of preoperative psychological assessment in the identification of the optimal treatment for patients with morbid obesity. 
A

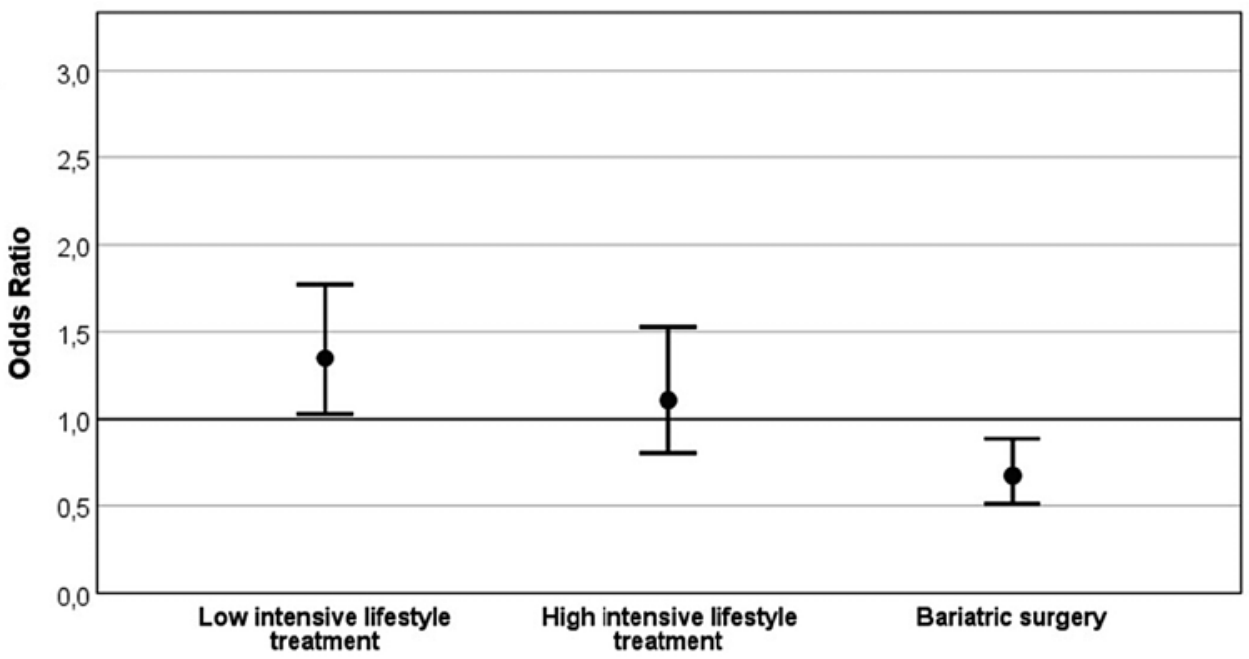

B
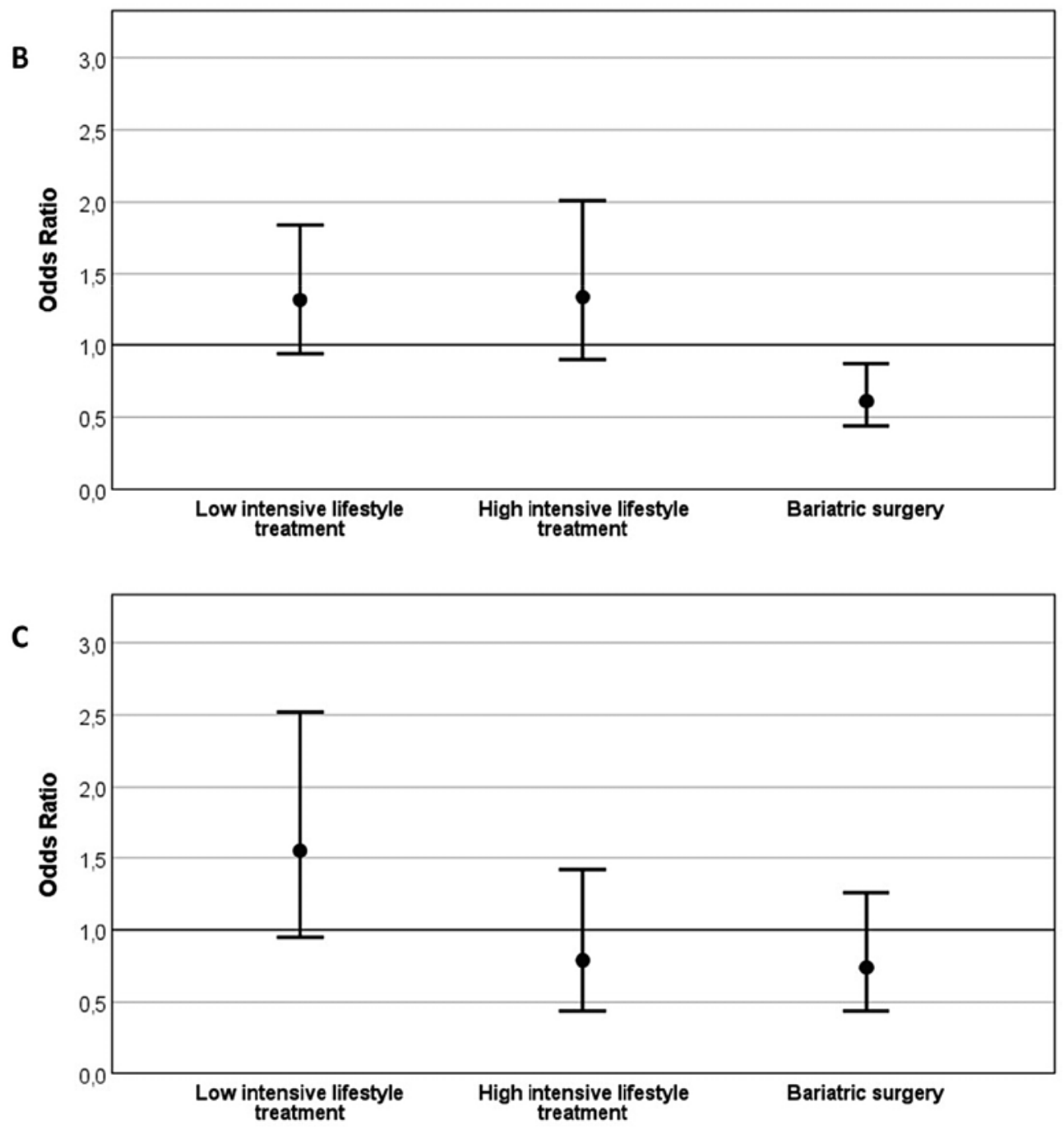

Fig. 3. Odds ratio for lifetime adversity in prediction subsequent obesity treatment. A All patients. B Female gender. C Male gender. 
Rønningen et al.: Lifetime Adversity Predicts Treatment Choice for Morbid Obesity

\section{The Role of Psychological Variables in Obesity Treatment}

To the best of our knowledge, this is the first study to assess the impact of lifetime adversity on treatment choice for morbid obesity. As suspected we found a high prevalence of lifetime adversity among patients with morbid obesity. The prevalence of lifetime adversity in the general population varies between 5 and $25 \%$, depending on the definition used [1721]. One large population study of more than 9,000 participants interviewed at 45 years of age showed that risk of adult obesity increased by $20-50 \%$ for several adversities in childhood [18]. Also, a Norwegian population study showed a general trend of dose-response association between lifestyle-related conditions (i.e. obesity and diabetes mellitus) and increasing number of childhood difficulties [21]. Our study partly confers with the results of another study, suggesting that surgical attrition developed secondary to psychological barriers [22]. The authors suggested that other factors than routinely reported as surgical motivators, including obesity-related comorbidities, might play a role in surgical attrition [22]. Moreover, given that bariatric surgery often is considered a stigmatized treatment option, patients' perceptions of weight stigma might have increased the psychological distress related to lifetime adversity in the clinical assessment of morbid obesity [23, 24]. On the other hand, a recent review update of the literature furthermore showed that fewer previous dieting attempts were the most consistent predictor of successful weight loss [25]. The authors argued that possible explanations might be related to a vulnerable psychological profile characterized by poor self-concept, body image disparagement, pessimistic attributions, and feelings of helplessness. Our results may also indicate that patients with severe obesity reporting lifetime adversity might benefit from a collaborative approach with their healthcare providers to identify the right long-term treatment option. As such, it is essential to explore the impact of adverse experiences prior to the onset of weight gain on eating habits, physical activity, social life, and emotional reactions. Furthermore, listening to patients' experiences and tailoring the best possible intervention programs has also been recommended as best practice $[9,26]$. The results of our study nevertheless underscore the importance of psychological evaluation in the presurgical assessments of patients with morbid obesity and indicate that adverse life experiences might play a role in the patients' treatment choice for obesity.

The present study showed a moderate overlap between lifetime adversity and symptoms indicative of psychiatric disease (i.e. self-reported anxiety or depression or established psychiatric illness), suggesting that patients with morbid obesity might have different psychological problems than patients with known psychiatric conditions. Our results indicate that focusing on lifetime adversity might identify patients with different psychological issues than patients with anxiety or depression. Consequently, patients who report lifetime adversity might need a different approach in the multidisciplinary work-up to identify the optimal treatment for each individual. Moreover, the patients who report lifetime adversity might benefit from a psychological evaluation to increase self-empowerment prior to intensive obesity treatment (i.e. high-intensive lifestyle treatment or bariatric surgery). Given the lack of consensus on therapeutic consequences of psychological evaluation, we suggest that a holistic psychosocial approach in the general assessment for patients with morbid obesity.

A previous study reported a positive association between increasing number of categories of adverse childhood exposure and severe obesity (BMI $\geq 35$ ), with a two-fold increased risk of developing severe obesity with 4 or more adverse childhood exposures [27]. Furthermore, a US integrated healthcare delivery system, including modifying environment in communities and schools, has been developed for the prevention and treatment of patients with obesity [28]. High internalized weight bias might be a barrier to weight management $[24,29]$. Also, negative feelings and shame about one's weight may prevent individuals from engaging in health promoting behaviors. Emotional eating and binge eating are furthermore reported more frequently in women than in men [30]. Internalized weight stigma and 
emotional eating patterns thus represent possible explanations for the difference in selfreported lifetime adversity by gender in subgroup analysis. Gender differences in pretreatment levels of weight control self-efficacy, binge eating and depressive symptoms have furthermore been reported as possible predictors of greater weight loss [31]. A previous study also showed vulnerability to "fat-stigma" in women's everyday relationships, suggesting a gender difference in social networking that might have impact on obesity treatment [32]. Our results underscore that gender differences should be considered in the psychosocial pretreatment of patients with morbid obesity.

Interestingly, great diversity was found among general practitioners' attitude towards health and factors affecting obesity in a recent Polish study examining 250 general practitioners [33]. Given the high prevalence of lifetime adversity among patients who refrained from intensive obesity treatment (i.e. chose low-intensive lifestyle treatment or bariatric surgery), we speculate if increased vigilance of lifetime adversity might have influenced the treatment choice of patients who reported lifetime experience. As such, the caretaker's perception of health might increase patients' psychological resilience, and facilitate the ability to endure the long term course of lifestyle intervention to obtain sustainable weight loss. Nevertheless, a recent review advocates that lack of recognition of obesity as a chronic disease has reduced the effect of evidence-based prevention and treatment [34]. Future studies are required to assess if exploring psychological variables perceived to be beneficial of obesity (and thus contribute to the reduced efficacy seen from lifestyle intervention in patients with obesity) will result in long-term weight loss.

\section{BMI, Obesity-Related Comorbidities, and Psychological Health}

Obesity staging classification systems have been developed to help individualize treatment for patients with morbid obesity $[35,36]$. Nevertheless, components of the metabolic syndrome, such as BMI and DM2, remain predominant predictors for choosing bariatric surgery over non-surgical obesity treatment [8]. The results of our study thus cohere with previous studies, indicating that patients with comorbid metabolic disease may favor a surgical treatment option. Communication of the progressive nature of DM2 might thus have influenced the decision-making process for both patients and healthcare personnel involved. One study of 144 patients showed improvements in most domains in the Kings Obesity Staging Criteria, including self-reported psychological health, 14 months after bariatric surgery [35]. However, the relatively short follow-up period might have underestimated the long-term complications post-bariatric surgery (such as weight regain, gastrointestinal discomfort, and reactive postprandial hypoglycemia) that might have influenced psychological health. Improved quality of life correlated with about $10 \%$ weight loss 10 years after bariatric surgery in the Swedish Obese Subject (SOS) study, and only two-thirds of the surgically treated patients reach this limit of $10 \%$ weight loss 10 years after bariatric surgery [13]. Thus, the impact of bariatric surgery on psychological health thus remains unknown.

Evidence suggests that long-term stress may affect biology, including brain structure and neuroendocrine mechanisms $[37,38]$. Also, plasticity genes have been associated with differential susceptibility to the development of obesity, i.e. the response to experience-based changes in cognitive, emotional and/or behavioral phenotypes [39]. Psychological stress has furthermore been associated with the development of metabolic syndrome and its components in prospective studies $[19,40]$. The role of bariatric surgery in resetting biological alterations associated with long-term stress exposure is however not clear, and the potential mechanisms for weight regain after bariatric surgery remain unknown. Consequently, in patients who report unresolved lifetime adversity, bariatric surgery alone might not result in sustainable weight loss and improved long-term health regardless of co-existing metabolic morbidities such as diabetes mellitus and hypertension. Future prospective studies are 
Rønningen et al.: Lifetime Adversity Predicts Treatment Choice for Morbid Obesity

required to assess if holistic preoperative psychosocial treatment might prevent weight regain and reduce risk of psychological worsening postoperatively.

\section{Functional Capacity and Obesity Treatment}

There is substantial evidence on the impact of physical activity on general health and longevity [41]. However, increased levels of physical activity have moderate impact on weight loss [42]. Our results partly cohere with previous studies indicating that candidates for bariatric surgery have low levels of physical activity and that this might have influenced the treatment choice for bariatric surgery $[8,43]$. Increased levels of physical activity have been reported after bariatric surgery [44]. Nevertheless, a 6-week preoperative physical activity intervention program improved both physical and mental health as well as self-enjoyment and motivations compared with standard presurgical care in 75 patients ( $90 \%$ women) with mean BMI 45. In our study, patients who chose bariatric surgery were more likely to have joint pain than patients who chose low-intensive lifestyle treatment. Thus, joint pain might have resulted in low levels of physical activity prior to surgery and possibly influenced treatment choice for morbid obesity.

Interestingly, our results also showed that employment status was associated with choosing high-intensive lifestyle treatment in women. A recent US population study showed that employment status was inversely related to disabilities, including obesity, and that employed individuals with disabilities reported better general and mental health than individuals who were not employed [45]. Two previous studies failed to show improvement in working status after bariatric surgery $[8,35]$. We thus speculate if potential positive effects of maintaining working status on self-esteem and quality of life might have influenced treatment choice in working patients with morbid obesity. Given that this result was present only in the female population, self-reported lifetime adversity might explain the preference for non-surgical obesity treatment among working women. The confidence interval for the association between employment status and choosing high-intensive lifestyle treatment was however large, and the results must be interpreted with caution.

\section{Strengths and Limitations}

The major strength of this study is that a large cohort of treatment-seeking patients was consecutively included. Most of our patients were Caucasian, and the results may thus not be valid in populations of other ethnicities. During the study period, only orlistat was available as an obesity drug in Norway, and many patients had already used orlistat as obesity treatment at the time of referral. As such, medical treatment was not included as a treatment choice for obesity in this study. Data on lifetime adversity were retrieved in a clinical setting without a premade questionnaire in line with another study assessing adverse life experiences in severely obese patients [46]. Thus, we cannot be sure that the definition of lifetime adversity is consistent. However, the registration was made by trained personnel, and only three different therapists retrieved the data throughout the study period. Also, when in doubt, the patients were discussed in the multidisciplinary team to assure appropriate interpretation of data. Furthermore, the prevalence of lifetime adversity was consistently reported throughout the study period of 4 years (data not shown). Given that the lifetime adversity was selfreported, our results might have underestimated the overall prevalence of lifetime adversity. Nevertheless, our results confer a clinical suspicion of a high prevalence of adverse events in patients with morbid obesity. Also, we cannot exclude the possibility that focusing on lifetime adversity in dialog with the patients might have influenced patient's treatment choice in this cross-sectional study. Only first-time referrals were included in this cross-sectional study without considering final treatment decisions from later referrals. Some patients used psychoactive drugs for sleeping disorders without confirmed psychiatric history, and the prevalence 
of psychiatric illness might thus have been overestimated. Finally, given the observational design, this study may not provide any causal relationship between lifetime adversity and obesity treatment.

\section{Conclusion}

This study shows that the prevalence of self-reported lifetime adversity is high among patients with morbid obesity. Also, in addition to increasing BMI, having DM2 and joint pain, lifetime adversity was associated with the patients' treatment choice for morbid obesity. Although multidisciplinary assessment for morbid obesity reveals no psychiatric illness, inherent psychological factors such as self-reported lifetime adversity might have major impact on an individual's health. Consequently, we suggest that a psychosocial approach is applied to assess possible lifetime adversity and to help find the treatment that would provide the best health benefit for each individual.

\section{Acknowledgments}

We acknowledge the contribution of Iren Pedersen at the Section for Morbid Obesity at Akershus University Hospital HF for the persistent effort on data collection. We would also like to thank Professor Anna Luise Kirkengen for valuable advice in editing the manuscript.

\section{Author Contributions}

T.G.V. designed the study. R.R., T.G.V., and A.C.P.W. collected the data. T.G.V. analyzed the data. All authors contributed to the interpretation of the data. R.R. and T.G.V. drafted the manuscript. A.C.P.W., R.R., N.H.G., and T.G.V. reviewed and edited the manuscript. All authors gave their final approval of the final version to be published.

\section{Disclosure Statement}

The authors declare that they have no competing interest.

\section{References}

1 Pischon T, Boeing H, Hoffmann K, Bergmann M, Schulze MB, Overvad K, et al. General and abdominal adiposity and risk of death in Europe. N Engl J Med. 2008 Nov;359(20):2105-20.

2 Whitlock G, Lewington S, Sherliker P, Clarke R, Emberson J, Halsey J, et al.; Prospective Studies Collaboration. Body-mass index and cause-specific mortality in 900000 adults: collaborative analyses of 57 prospective studies. Lancet. 2009 Mar;373(9669):1083-96.

3 Aftab H, Risstad H, Søvik TT, Bernklev T, Hewitt S, Kristinsson JA, et al. Five-year outcome after gastric bypass for morbid obesity in a Norwegian cohort. Surg Obes Relat Dis. 2014 Jan-Feb;10(1):71-8.

4 Jakobsen GS, Småstuen MC, Sandbu R, Nordstrand N, Hofsø D, Lindberg M, et al. Association of Bariatric Surgery vs Medical Obesity Treatment with Long-term Medical Complications and Obesity-Related Comorbidities. JAMA. 2018 Jan;319(3):291-301.

5 King WC, Chen JY, Courcoulas AP, Dakin GF, Engel SG, Flum DR, et al. Alcohol and other substance use after bariatric surgery: prospective evidence from a U.S. multicenter cohort study. Surg Obes Relat Dis. 2017 Aug; 13(8):1392-402.

6 Acevedo MB, Eagon JC, Bartholow BD, Klein S, Bucholz KK, Pepino MY. Sleeve gastrectomy surgery: when 2 alcoholic drinks are converted to 4. Surg Obes Relat Dis. 2018 Mar;14(3):277-83.

7 Padwal RS, Pajewski NM, Allison DB, Sharma AM. Using the Edmonton obesity staging system to predict mortality in a population-representative cohort of people with overweight and obesity. CMAJ. 2011 Oct; 183(14):E1059-66. 
8 Valderhaug TG, Aasheim ET, Sandbu R, Jakobsen GS, Småstuen MC, Hertel JK, et al. The association between severity of King's Obesity Staging Criteria scores and treatment choice in patients with morbid obesity: a retrospective cohort study. BMC Obes. 2016 Dec;3(1):51.

9 Torti J, Luig T, Borowitz M, Johnson JA, Sharma AM, Campbell-Scherer DL. The 5As team patient study: patient perspectives on the role of primary care in obesity management. BMC Fam Pract. 2017 Feb;18(1):19.

10 Kaplan LM, Golden A, Jinnett K, Kolotkin RL, Kyle TK, Look M, et al. Perceptions of Barriers to Effective Obesity Care: Results from the National ACTION Study. Obesity (Silver Spring). 2018 Jan;26(1):61-9.

11 World Medical Association Declaration of Helsinki. World Medical Association Declaration of Helsinki: ethical principles for medical research involving human subjects. JAMA. 2000 Dec;284(23):3043-5.

12 Alberti KG, Eckel RH, Grundy SM, Zimmet PZ, Cleeman JI, Donato KA, et al.; International Diabetes Federation Task Force on Epidemiology and Prevention; Hational Heart, Lung, and Blood Institute; American Heart Association; World Heart Federation; International Atherosclerosis Society; International Association for the Study of Obesity. Harmonizing the metabolic syndrome: a joint interim statement of the International Diabetes Federation Task Force on Epidemiology and Prevention; National Heart, Lung, and Blood Institute; American Heart Association; World Heart Federation; International Atherosclerosis Society; and International Association for the Study of Obesity. Circulation. 2009 Oct;120(16):1640-5.

13 Karlsson J, Taft C, Rydén A, Sjöström L, Sullivan M. Ten-year trends in health-related quality of life after surgical and conventional treatment for severe obesity: the SOS intervention study. Int J Obes. 2007 Aug; 31(8):1248-61.

14 Luppino FS, de Wit LM, Bouvy PF, Stijnen T, Cuijpers P, Penninx BW, et al. Overweight, obesity, and depression: a systematic review and meta-analysis of longitudinal studies. Arch Gen Psychiatry. 2010 Mar;67(3):220-9.

15 Gade H, Friborg O, Rosenvinge JH, Småstuen MC, Hjelmesæth J. The Impact of a Preoperative Cognitive Behavioural Therapy (CBT) on Dysfunctional Eating Behaviours, Affective Symptoms and Body Weight 1 Year after Bariatric Surgery: A Randomised Controlled Trial. Obes Surg. 2015 Nov;25(11):2112-9.

16 Pataky Z, Carrard I, Golay A. Psychological factors and weight loss in bariatric surgery. Curr Opin Gastroenterol. 2011 Mar;27(2):167-73.

17 Andenæs R, Helseth S, Misvær N, Småstuen MC, Ribu L. Psychosocial factors are strongly associated with insomnia in users and nonusers of prescribed sleep medication: evidence from the HUNT3 study. J Multidiscip Healthc. 2016 Oct; 9:547-55.

18 Thomas C, Hyppönen E, Power C. Obesity and type 2 diabetes risk in midadult life: the role of childhood adversity. Pediatrics. 2008 May;121(5):e1240-9.

19 Rutters F, Pilz S, Koopman AD, Rauh SP, Pouwer F, Stehouwer CD, et al. Stressful life events and incident metabolic syndrome: the Hoorn study. Stress. 2015;18(5):507-13.

20 Church C, Andreassen OA, Lorentzen S, Melle I, Aas M. Childhood Trauma and Minimization/Denial in People with and without a Severe Mental Disorder. Front Psychol. 2017 Aug;8:1276.

21 Tomasdottir MO, Sigurdsson JA, Petursson H, Kirkengen AL, Krokstad S, McEwen B, et al. Self Reported Childhood Difficulties, Adult Multimorbidity and Allostatic Load. A Cross-Sectional Analysis of the Norwegian HUNT Study. PLoS One. 2015 Jun;10(6):e0130591.

22 Mahony D. Bariatric surgery attrition secondary to psychological barriers. Clin Obes. 2013 Feb;3(1-2):32-8.

23 Vartanian LR, Fardouly J. Reducing the stigma of bariatric surgery: benefits of providing information about necessary lifestyle changes. Obesity (Silver Spring). 2014 May;22(5):1233-7.

24 Hayward LE, Vartanian LR, Pinkus RT. Weight Stigma Predicts Poorer Psychological Well-Being through Internalized Weight Bias and Maladaptive Coping Responses. Obesity (Silver Spring). 2018 Apr;26(4):755-61.

25 Carraça EV, Santos I, Mata J, Teixeira PJ. Psychosocial Pretreatment Predictors of Weight Control: a Systematic Review Update. Obes Facts. 2018;11(1):67-82.

26 Yumuk V, Tsigos C, Fried M, Schindler K, Busetto L, Micic D, et al.; Obesity Management Task Force of the European Association for the Study of Obesity. European Guidelines for Obesity Management in Adults. Obes Facts. 2015;8(6):402-24.

27 Felitti VJ, Anda RF, Nordenberg D, Williamson DF, Spitz AM, Edwards V, et al. Relationship of childhood abuse and household dysfunction to many of the leading causes of death in adults. The Adverse Childhood Experiences (ACE) Study. Am J Prev Med. 1998 May;14(4):245-58.

28 Tsai AG, Histon T, Donahoo WT, Hashmi S, Murali S, Latare P, et al. Investing in Obesity Treatment: Kaiser Permanente's Approach to Chronic Disease Management. Curr Obes Rep. 2016 Sep;5(3):307-11.

29 Carels RA, Young KM, Wott CB, Harper J, Gumble A, Hobbs MW, et al. Internalized weight stigma and its ideological correlates among weight loss treatment seeking adults. Eat Weight Disord. 2009 Jun-Sep;14(23):e92-7.

30 Larsen JK, van Strien T, Eisinga R, Engels RC. Gender differences in the association between alexithymia and emotional eating in obese individuals. J Psychosom Res. 2006 Mar;60(3):237-43.

31 Presnell K, Pells J, Stout A, Musante G. Sex differences in the relation of weight loss self-efficacy, binge eating, and depressive symptoms to weight loss success in a residential obesity treatment program. Eat Behav. 2008 Apr;9(2):170-80.

32 Brewis AA, Hruschka DJ, Wutich A. Vulnerability to fat-stigma in women's everyday relationships. Soc Sci Med. 2011 Aug; 73(4):491-7. 
Rønningen et al.: Lifetime Adversity Predicts Treatment Choice for Morbid Obesity

33 Bąk-Sosnowska M, Skrzypulec-Plinta V. Health behaviors, health definitions, sense of coherence, and general practitioners' attitudes towards obesity and diagnosing obesity in patients. Arch Med Sci. 2017 Mar;13(2): 433-40.

34 Sharma AM, Ramos Salas X. Obesity Prevention and Management Strategies in Canada: Shifting Paradigms and Putting People First. Curr Obes Rep. 2018 Jun;7(2):89-96.

35 Aasheim ET, Aylwin SJ, Radhakrishnan ST, Sood AS, Jovanovic A, Olbers T, et al. Assessment of obesity beyond body mass index to determine benefit of treatment. Clin Obes. 2011 Apr;1(2-3):77-84.

36 Sharma AM, Kushner RF. A proposed clinical staging system for obesity. Int J Obes. 2009 Mar;33(3):289-95.

37 Teicher MH, Samson JA, Anderson CM, Ohashi K. The effects of childhood maltreatment on brain structure, function and connectivity. Nat Rev Neurosci. 2016 Sep;17(10):652-66.

38 McEwen BS. Redefining neuroendocrinology: epigenetics of brain-body communication over the life course. Front Neuroendocrinol. 2018 Apr;49:8-30.

39 Dalle Molle R, Fatemi H, Dagher A, Levitan RD, Silveira PP, Dubé L. Gene and environment interaction: is the differential susceptibility hypothesis relevant for obesity? Neurosci Biobehav Rev. 2017 Feb;73(Feb):326-39.

40 Bergmann N, Gyntelberg F, Faber J. The appraisal of chronic stress and the development of the metabolic syndrome: a systematic review of prospective cohort studies. Endocr Connect. 2014 Jun;3(2):R55-80.

41 Ekelund U, Steene-Johannessen J, Brown WJ, Fagerland MW, Owen N, Powell KE, et al.; Lancet Physical Activity Series 2 Executive Committe; Lancet Sedentary Behaviour Working Group. Does physical activity attenuate, or even eliminate, the detrimental association of sitting time with mortality? A harmonised meta-analysis of data from more than 1 million men and women. Lancet. 2016 Sep;388(10051):1302-10.

42 Swift DL, Johannsen NM, Lavie CJ, Earnest CP, Church TS. The role of exercise and physical activity in weight loss and maintenance. Prog Cardiovasc Dis. 2014 Jan-Feb;56(4):441-7.

43 Bond DS, Jakicic JM, Vithiananthan S, Thomas JG, Leahey TM, Sax HC, et al. Objective quantification of physical activity in bariatric surgery candidates and normal-weight controls. Surg Obes Relat Dis. 2010 Jan-Feb;6(1): 72-8.

44 Bond DS, Jakicic JM, Unick JL, Vithiananthan S, Pohl D, Roye GD, et al. Pre- to postoperative physical activity changes in bariatric surgery patients: self report vs. objective measures. Obesity (Silver Spring). 2010 Dec; 18(12):2395-7.

45 Reichard A, Stransky M, Brucker D, Houtenville A. The relationship between employment and health and health care among working-age adults with and without disabilities in the United States. Disabil Rehabil. 2018 May 20:1-9.

46 Gabert DL, Majumdar SR, Sharma AM, Rueda-Clausen CF, Klarenbach SW, Birch DW, et al. Prevalence and predictors of self-reported sexual abuse in severely obese patients in a population-based bariatric program. J Obes. 2013;2013:374050. 